\title{
A systematic review and meta-analysis of the comparative curative effects of warm acupuncture and other traditional Chinese medicines in the treatment of knee osteoarthritis
}

\author{
Si'an Jin, Xuefeng Guan \\ Liaoning University of Traditional Chinese Medicine, Shenyang, China \\ Contributions: (I) Conception and design: Both authors; (II) Administrative support: Both authors; (III) Provision of study materials or patients: \\ Both authors; (IV) Collection and assembly of data: Both authors; (V) Data analysis and interpretation: Both authors; (VI) Manuscript writing: Both \\ authors; (VII) Final approval of manuscript: Both authors. \\ Correspondence to: Xuefeng Guan. Liaoning University of Traditional Chinese Medicine, 79 Chongshan East Road, Huanggu District, Shenyang \\ 110000, China. Email: lnzygxf@163.com.
}

\begin{abstract}
Background Knee osteoarthritis (KOA) is more common in middle-aged and elderly people, and seriously affects the quality of life of those affected. Traditional Chinese medicine (TCM) treatment of KOA has been widely recognized. In recent years, warm needling acupuncture (WNA) has been used to treat KOA and has achieved good results. However, there is a lack of comparison of the efficacy of WNA and other TCM treatments for KOA.

Methods: We conducted a search for reports of WNA and/or TCM treatment of KOA in English- and Chinese-language databases. The data was retrieved from inception of the database until October 2021. The Cochrane risk of bias tool was used to evaluate the quality of the included studies, and the network metaanalysis was performed using the software RevMan 5.20.

Results: A total of 8 articles met the inclusion criteria, including 399 patients treated with WNA (WNA group), and 396 patients treated with other TCM (TCM group). The results of meta-analysis showed that compared with patients in the TCM group, the effective rate [relative risk (RR)] was 1.18, 95\% confidence interval (CI): 1.06 to 1.33, the last follow-up osteoarthritis index [mean difference (MD)] was $-6.93,95 \%$ CI: -12.14 to -1.72 , and the last follow-up knee pain visual analogue scale (VAS) MD was $-1.06,95 \%$ CI: -1.61 to -0.51 , which were all statistically significant. However, the difference in daily activities (MD: $-4.31,95 \%$ CI: -10.90 to 2.28 ) was not statistically significant.

Discussion: Compared with other TCM treatments for KOA, WNA has better overall patient efficacy. However, further randomized controlled studies are needed to compare WNA and other TCM treatments individually to confirm the efficacy of WNA.
\end{abstract}

Keywords: Warm needling acupuncture (WNA); traditional Chinese medicine (TCM); knee osteoarthritis (KOA); meta-analysis

Submitted Dec 12, 2021. Accepted for publication Jan 30, 2022.

doi: 10.21037/apm-21-3972

View this article at: https://dx.doi.org/10.21037/apm-21-3972

\section{Introduction}

Knee osteoarthritis (KOA) is a common disease in degenerative osteoarthritis, which is more common in the elderly. As knee osteoarthritis can cause knee joint function limitation and pain, the disease seriously affects the quality of life and work of patients. Moreover, KOA is one of the main diseases that cause knee dysfunction and disability in the elderly $(1,2)$. With the continuous intensification of 
the aging of the world population, the incidence of KOA is increasing annually (3). The development of KOA is often related to many factors, including the patient's age, gender, knee joint trauma, obesity, inflammation, occupational labor intensity, exercise intensity, and genetics (4).

The main pathological features of KOA are loss of articular cartilage and subchondral bone sclerosis (5). In the early stage of KOA, conservative treatments are provided, including health education, activity adjustment, physical therapy, weight loss, and so on. When conservative treatment fails, treatment involves the use of antiinflammatory and/or analgesics, intra-articular hyaluronic acid and/or steroid injections, and arthroscopic lavage. The main treatment for advanced moderate to severe pain and/ or disability is total knee arthroplasty (6). Warm needle acupuncture (WNA) is a characteristic treatment method of traditional Chinese medicine (TCM). WNA is to add moxa at the tail of the needle after the needle is punctured. After ignition, the heat energy can be transmitted to the body through the needle body, so as to achieve the effect of preventing and treating diseases. With the expansion of research, results have shown that WNA can achieve the purpose disease curation by regulation of blood circulation, nervous system, and even immune function (7). At present, WNA is widely used to treat KOA in China, and its curative effect is equal to or better than that of acupuncture alone.

Encompassing the modalities of acupuncture, moxibustion, herbal medicine, and massage, TCM has been used in China for thousands of years to treat many diseases (7-9). These methods have been implemented worldwide, mainly as adjuvant treatments for chronic pain and diseases (such as diabetes and cancer) (8-11). There are a variety of TCM treatments that can be used to treat KOA. Studies have shown that TCM is an effective treatment method that can relieve pain and improve knee joint function in patients with $\operatorname{KOA}(12,13)$. Among them, fumigation and washing therapy is the most commonly used treatment method, which has been shown to have definite effects (14).

Having been reported as having good efficacy in the treatment of KOA, WNA has attracted increasing attention, but no comparison has been made regarding its efficacy compared with other TCM treatment methods. Therefore, this study aimed to analyze the efficacy of WNA and other TCM treatments for KOA. We present the following article in accordance with the PRISMA reporting checklist (available at https://apm.amegroups.com/article/ view/10.21037/apm-21-3972/rc).

\section{Methods}

\section{Search strategy}

We used the search terms "Warm Needle Acupuncture" and "Knee Osteoarthritis" or "Warm Needle Acupuncture" and "Degenerative Knee Osteoarthropathy" or "Warm Needle Acupuncture" and "Knee Osteoarthritis" or "WNA" And "KOA", and "traditional Chinese medicine" and "knee osteoarthritis" or "traditional Chinese medicine" and "degenerative knee osteoarthritis" or "traditional Chinese medicine" and "knee osteoarthritis" or "TCM" and "KOA" to search the English biomedical databases of PubMed, Cochrane Central Register of Controlled Trials, Embase, Web of Science, and major Chinese biomedical databases such as China National Knowledge Infrastructure (CNKI), Wanfang, and Weipu. The retrieval time was from the establishment of the database to October 2021.

\section{Inclusion and exclusion criteria}

The inclusion criteria were as follows: (I) patient aged 45 years and above; (II) clinically diagnosed with KOA; (III) treatment was WNA or other TCM treatment methods; (IV) or comparison of WNA and TCM efficacy of treating $\mathrm{KOA}$; $(\mathrm{V})$ number of reported patient cases was at least 10; (VI) outcome indicators included overall effective rate, daily activities after treatment, last follow-up osteoarthritis index scale, and last follow-up knee visual analogue scale for pain (VAS). The exclusion criteria were as follows: (I) patient was younger than 45 years; (II) KOA treated by surgery; (III) reports with the same author and data (duplicate); (IV) research reports on non-TCM or WNA treatment; (V) reports that did not describe the indicators required for the study.

\section{Paper screening and quality assessment}

Authors who had been trained to independently complete article screening independently evaluated the title and abstract of each article to determine whether it was eligible for inclusion in the study. If the article appeared to meet the required standards for the study, the investigator then reviewed the full text to decide upon its inclusion. If reviewers disagreed on the eligibility of an article, they decided upon its inclusion in the study through discussion. 
If the discussion failed to reach a consensus, a third reviewer was invited to arbitrate. The Cochrane risk of bias tool (https://methods.cochrane.org/bias/resources/ rob-2-revised-cochrane-risk-bias-tool-randomized-trials) was used to assess the quality and risk of bias of included articles. Evaluation items included the following: random sequence generation, allocation concealment, blinding of investigators and participants, blinded evaluation of research outcomes, completeness of outcome data, selective outcome reports, and other biases. According to the evaluation, the biases are classified into high-risk, low risk, and unclear. The 2 reviewers carried out the above process separately, and if there was any difference in their findings, a consensus was reached through discussion.

\section{Data extraction}

The included articles are grouped according to WNA treatment and other TCM treatments. After further reading through the article, the included patients were divided into a WNA group and other TCM groups. The data of each article was also independently extracted by 2 reviewers, and entered in the form for later use. The extracted data included the author's name, country, publication time, journal name, number of participants, effective rate, daily exercise, last follow-up osteoarthritis index scale, VAS, and so on. After the data was extracted, the 2 reviewers discussed and unified the differences in the data.

\section{Statistical analysis}

Percentages and relative risk (RR) or mean difference (MD) with $95 \%$ confidence interval $(\mathrm{CI})$ were used to describe the data. The $\mathrm{I}^{2}$ test was used to test the heterogeneity. If the heterogeneity between studies was small $\left(\mathrm{P}>0.1, \mathrm{I}^{2}<50 \%\right)$, the fixed-effects model was used to merge the effect sizes; if the heterogeneity between studies was obvious $\left(\mathrm{P} \leq 0.1, \mathrm{I}^{2} \geq 50 \%\right)$, the random-effects model was used to merge the effect size. And the sensitivity analysis was carried out according to the Cochrane systematic review method. Statistical analyses and graphs were performed using RevMan 5.20 (The Nordic Cochrane Center, The Cochrane Collaboration, Copenhagen, Denmark, 2012) software provided by Cochrane Collaboration. A P value $\leq 0.05$ was considered statistically significant. The funnel plot was used to analyze the risk of publication bias.

\section{Results}

\section{Search and screen results}

There were 590 records confirmed from the English and Chinese databases, 63 unsuitable records were removed, leaving 527 records, of which 246 records were excluded due to low quality, review, and case report designs. Finally, 152 articles were obtained after retrieval. After the final research and evaluation, 8 articles were retained to perform this meta-analysis. The specific process is shown in Figure 1.

\section{Study characteristics}

Among the 8 articles available, 8 reported on the effective rate, 2 reported on daily exercise, 6 reported on the last follow-up osteoarthritis index scale, and 6 articles reported on the last follow-up knee joint pain VAS. All selected articles had made a clear KOA diagnosis on the patient. There were 399 patients treated with WNA and 396 patients treated with TCM. The basic characteristics of the included articles are shown in Table 1.

All included articles describe random sequence generation, report allocation concealment, and use of double-blind methods for participants and personnel. Only 1 article reported the blinding of the outcome assessment, and the remaining 7 articles did not describe the use of the blinding of the outcome assessment. All articles described the risks of complete outcome data bias, selective reporting bias, and other biases. The evaluation report is shown in Figure 2.

\section{Risk of bias}

Regard random sequence generation bias and allocation concealment bias, all studies were classified as low-risk. The blinding bias of participants and researchers was assessed: 1 study was low risk (22), and the remaining 7 studies were high risk (15-21). Regarding outcome assessment blinding bias, 1 study was high-risk (22), and the remaining 7 studies were unclear (15-21). For incomplete outcome data bias, selective reporting domain bias, and other biases, all studies were assessed as low-risk. The specific report is shown in Figure 3.

\section{Meta-analysis results}

Effective rate of WNA and other TCM treatment KOA After screening according to the pre-established criteria, we 


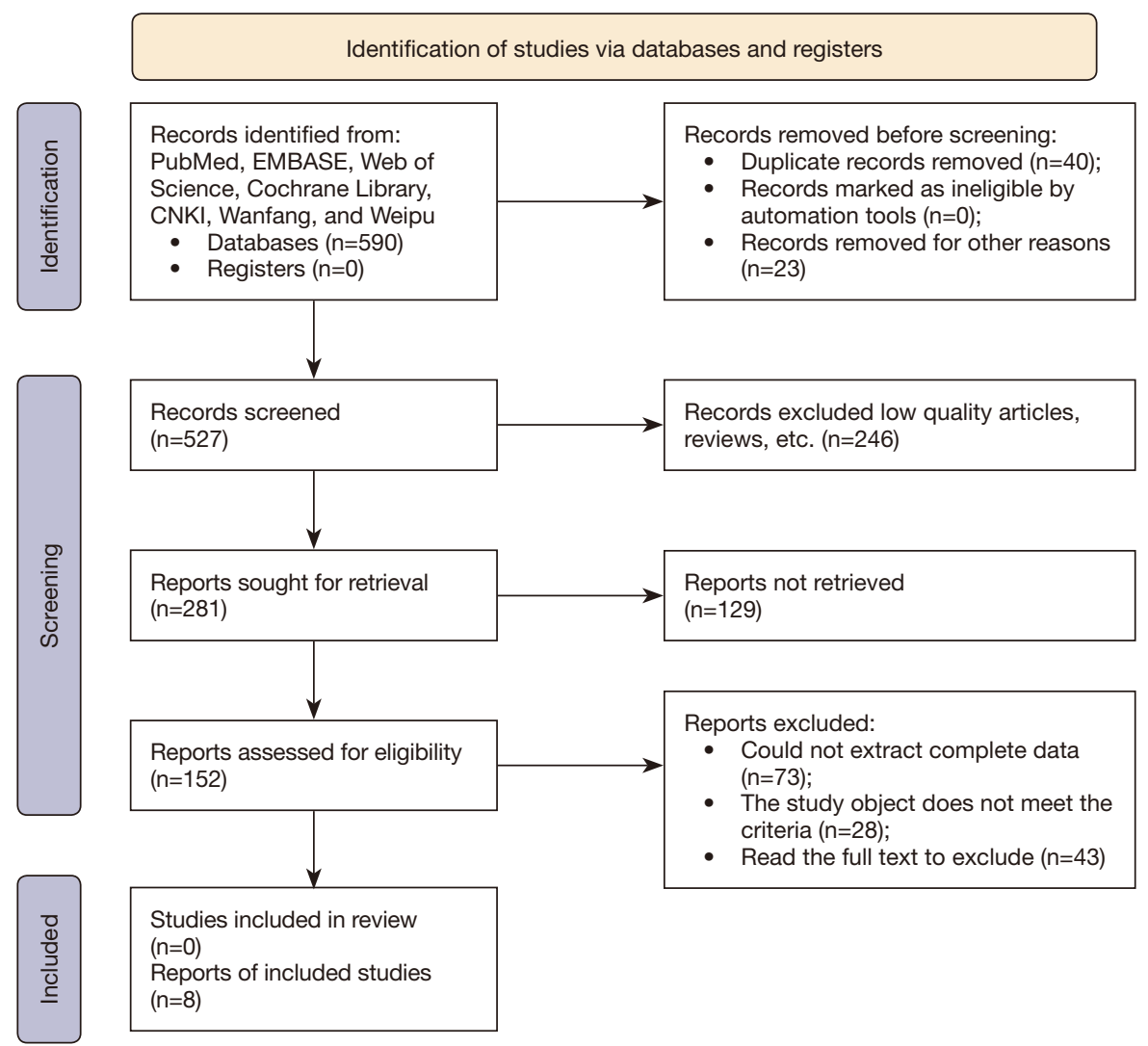

Figure 1 Flow diagram of the search, screening, and inclusion process.

Table 1 Basic characteristics of the study articles

\begin{tabular}{lcclcc}
\hline Author & Country & Year & Journal & WNA group (n) & TCM group (n) \\
\hline Gao et al. (15) & China & 2012 & Zhongguo Zhen Jiu & 35 & 34 \\
Li et al. (16) & China & 2006 & Zhongguo Zhen Jiu & 56 & 24 \\
Han et al. (17) & China & 2018 & Henan Traditional Chinese Medicine & 24 & 30 \\
Mo et al. (18) & China & 2015 & Guangzhou University of Chinese Medicine & 30 & 30 \\
Pan et al. (19) & China & 2014 & Shandong University of Traditional Chinese Medicine & 40 & 40 \\
Pan et al. (20) & China & 2020 & Chinese Journal of General Practice & 34 \\
Qu et al. (21) & China & 2015 & Journal of Acupuncture and Tuina Science & 150 \\
Wu et al. (22) & China & 2009 & Journal of Acupuncture and Tuina Science & 150 \\
\hline
\end{tabular}

WNA, warm needle acupuncture; TCM, traditional Chinese medicine.

obtained 8 articles which reported on the effective rate, and analyzed the efficacy of WNA and other TCM modalities in the treatment of KOA. These articles included cumulative totals of 399 WNA-treated patients and 396 other TCMtreated patients. The analysis results yielded $\mathrm{P}=0.0004$ and
$\mathrm{I}^{2}=74 \%$, indicating that there was heterogeneity between the effective rates, so the random effects model was used for joint analysis. The combined effect size RR was $1.18,95 \%$ CI: 1.06 to 1.33, as shown in Figure 4. The overall effect size test was $\mathrm{Z}=2.89, \mathrm{P}=0.004$, so the meta-analysis results 


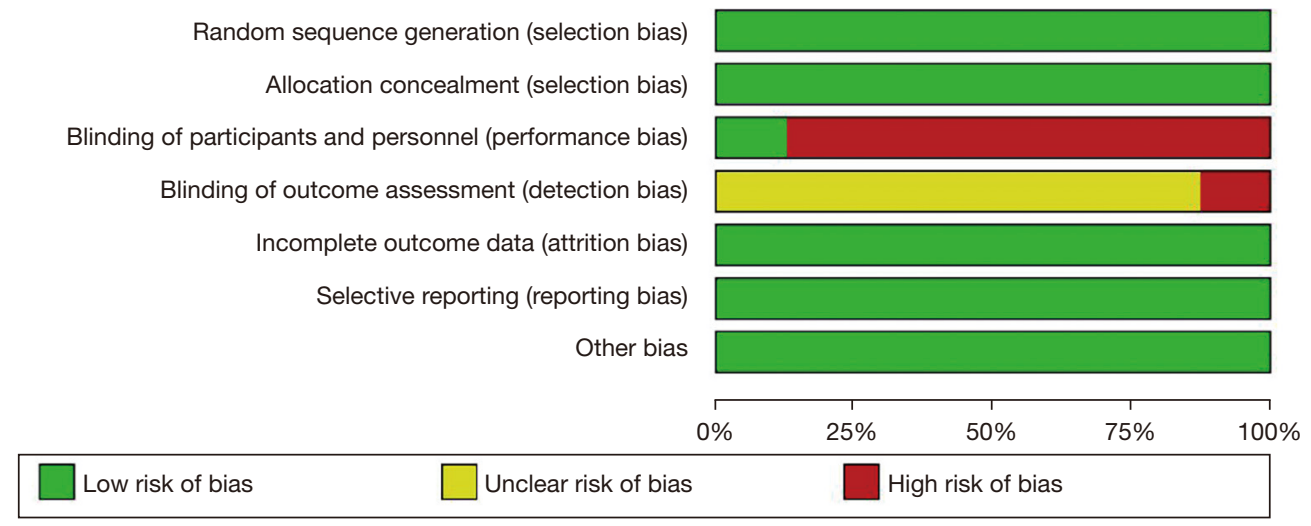

Figure 2 The intensity and distribution of the quality risk of the articles included in the study.

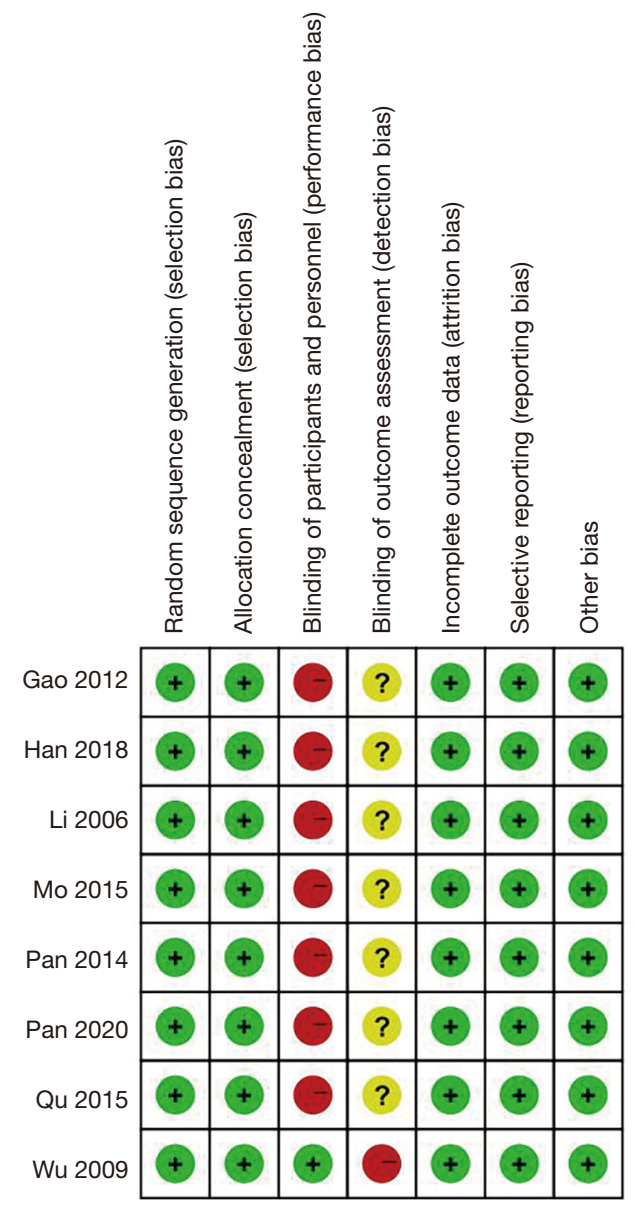

Figure 3 Literature quality evaluation details.

suggested that the effective rate of the WNA treatment group was statistically different from that of the other TCM treatment groups.

\section{Effect of WNA and other TCM treatment on KOA patient daily activities}

After screening, 2 reports on daily activities were obtained, which investigated the daily activities of WNA and other TCMs after treatment of KOA. They 2 articles included 65 WNA-treated patients and 64 other TCM-treated patients. The analysis results revealed that $\mathrm{P}=0.03$ and $\mathrm{I}^{2}=79 \%$, indicating that there was heterogeneity between daily activities, therefore, the random effects model was used for combined analysis. The combined effect size MD was -4.31 , 95\% CI: -10.90 to 2.28, as shown in Figure 5. The overall effect size test was $Z=1.28, P=0.20$, so the meta-analysis results suggested that there was no statistical difference in daily activities between the WNA treatment group and the other TCM treatment group.

\section{WNA and other TCM treatment of KOA last follow- up osteoarthritis index scale}

After screening, we obtained 6 reports on the last followup osteoarthritis index, and analyzed the osteoarthritis index at the last follow-up after WNA and other TCM treatments for KOA. These 6 reports included a total of 215 WNA-treated patients and 212 other TCM-treated patients. The analysis results showed indicated that there was heterogeneity between the osteoarthritis index scales $\left(\mathrm{P}<0.00001, \mathrm{I}^{2}=96 \%\right)$, so the random effects model was used for combined analysis. The combined effect size MD was $-6.93,95 \%$ CI: -12.14 to -1.72 , as shown in Figure 6. The overall effect size test was $Z=2.61, P=0.009$, so the meta-analysis results suggested that there was a statistical difference between the WNA treatment group and the other TCM treatment groups in the osteoarthritis index scale. 


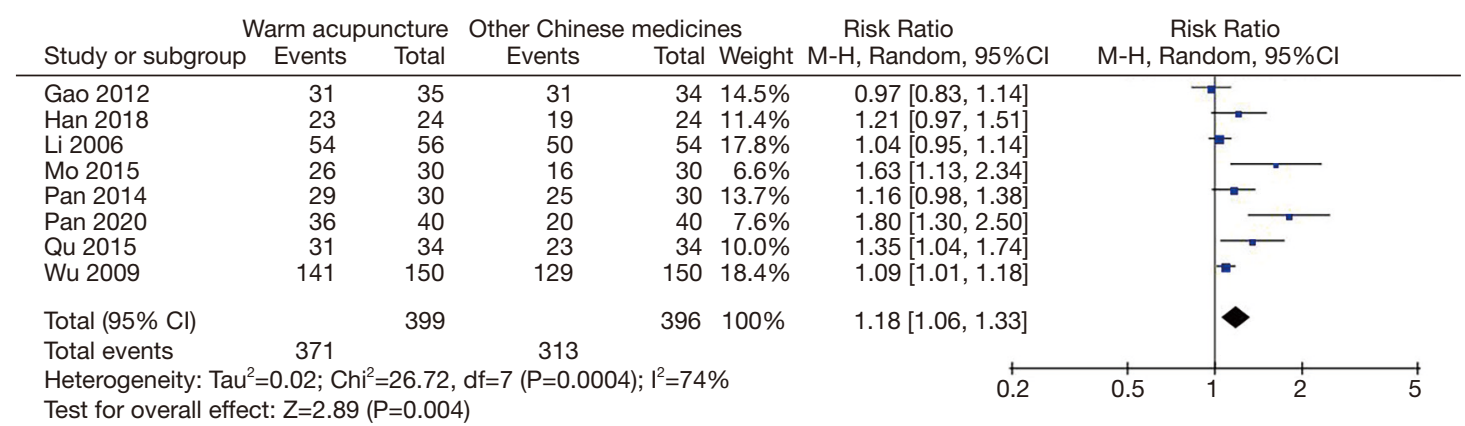

Figure 4 Forest plot of effective rate. Comparison of effective rate of KOA between the WNA group and the other TCM group. Statistical method: Mantel-Haenszel of random effects model (RR and 95\% CI). CI, confidence interval; KOA, knee osteoarthritis; WNA, warm needle acupuncture; TCM, traditional Chinese medicine; RR, relative risk.

\begin{tabular}{|c|c|c|c|c|c|c|c|c|c|c|}
\hline \multirow[b]{2}{*}{ Study or subgroup } & \multicolumn{3}{|c|}{ Warm acupuncture } & \multicolumn{4}{|c|}{ Other Chinese medicines } & \multirow{2}{*}{$\begin{array}{c}\text { Mean Difference } \\
\text { IV, Random, 95\%Cl }\end{array}$} & \multicolumn{2}{|l|}{ Mean Difference } \\
\hline & Mean & SD & Total & Mean & SD & Total & Weight & & IV, Random, $95 \% \mathrm{Cl}$ & \\
\hline Gao 2012 & 26.54 & 9.31 & 35 & 27.37 & 10.19 & 34 & $48.3 \%$ & $-0.83[-5.44,3.78]$ & & \\
\hline Pan 2014 & 8.17 & 6.23 & 30 & 15.73 & 8.94 & 30 & $51.7 \%$ & $-7.56[-11.46,-3.66]$ & $\mathbf{\square}$ & \\
\hline Total $(95 \% \mathrm{Cl})$ & & & 65 & & & 64 & $100 \%$ & $-4.31[-10.90,2.28]$ & & \\
\hline $\begin{array}{l}\text { Heterogeneity: Tau } \\
\text { Test for overall effe }\end{array}$ & $\begin{array}{l}1^{2}=17.90 \\
\text { ect: } Z=1\end{array}$ & $\begin{array}{l}; \mathrm{Chi}^{2}= \\
.28(\mathrm{P}=\end{array}$ & $\begin{array}{l}=4.77, \mathrm{c} \\
=0.20)\end{array}$ & $d f=1(P=$ & 3); & $79 \%$ & & -100 & -50 & 100 \\
\hline
\end{tabular}

Figure 5 Forest plot of daily activities. Comparison of daily activities between the WNA group and the other TCM group. Statistical method: inverse variance of the random effects model (MD and 95\% CI). CI, confidence interval; WNA, warm needle acupuncture; MD, mean difference.

\begin{tabular}{|c|c|c|c|c|c|c|c|c|c|c|}
\hline \multirow[b]{2}{*}{ Study or subgroup } & \multicolumn{3}{|c|}{ Warm acupuncture } & \multicolumn{4}{|c|}{ Other Chinese medicines } & \multirow{2}{*}{$\begin{array}{c}\text { Mean Difference } \\
\text { IV, Random, 95\% Cl }\end{array}$} & \multirow{2}{*}{$\begin{array}{l}\text { Mean Difference } \\
\text { IV, Random, } 95 \% \mathrm{Cl}\end{array}$} & \\
\hline & Mean & SD & Total & Mean & SD & Total & Weight & & & \\
\hline $\begin{array}{l}\text { Gao } 2012 \\
\text { Han } 2018 \\
\text { Li 2006 } \\
\text { Mo 2015 } \\
\text { Pan 2014 } \\
\text { Pan 2020 }\end{array}$ & $\begin{array}{r}49.28 \\
5.64 \\
4.57 \\
15.57 \\
13.57 \\
10.96\end{array}$ & $\begin{array}{r}11.71 \\
3.25 \\
1.88 \\
5.575 \\
8.85 \\
1.89\end{array}$ & $\begin{array}{l}35 \\
24 \\
56 \\
30 \\
30 \\
40\end{array}$ & $\begin{array}{r}50.79 \\
8.92 \\
7.55 \\
22.4 \\
23.53 \\
27.56\end{array}$ & $\begin{array}{r}12.68 \\
5.91 \\
2.4 \\
7.708 \\
10.67 \\
7.2\end{array}$ & $\begin{array}{l}34 \\
24 \\
54 \\
30 \\
30 \\
40\end{array}$ & $\begin{array}{l}14.8 \% \\
17.3 \% \\
18.0 \% \\
16.8 \% \\
15.6 \% \\
17.5 \%\end{array}$ & $\begin{array}{r}-1.51[-7.27,4.25] \\
-3.28[-5.98,-0.58] \\
-2.98[-3.79,-2.17] \\
-6.83[-10.23,-3.43] \\
-9.96[-14.92,-5.00] \\
-16.60[-18.91,-14.29]\end{array}$ & $\begin{array}{l}= \\
= \\
=\end{array}$ & \\
\hline Total $(95 \% \mathrm{Cl})$ & & & 215 & & & 212 & $100 \%$ & $-6.93[-12.14,-1.72]$ & 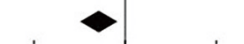 & \\
\hline $\begin{array}{l}\text { Heterogeneity: Ta } \\
\text { Test for overall eff }\end{array}$ & fect: $Z=$ & $\begin{array}{l}3 ; \mathrm{Cr} \\
2.61\end{array}$ & $\begin{array}{l}=127.5 \\
=0.00 \leqq\end{array}$ & 5 & -0.00 & ר1 & con & -5 & -25 & 50 \\
\hline
\end{tabular}

Figure 6 Forest plot of last follow-up osteoarthritis index scales. Comparison of last follow-up osteoarthritis index scales between the WNA group and the other TCM group. Statistical method: inverse variance of the random effects model (MD and 95\% CI). CI, confidence interval; WNA, warm needle acupuncture; MD, mean difference.

\section{WNA and other TCM treatment of KOA last follow-up VAS}

After screening, we obtained 6 reports on VAS at the last follow-up, and analyzed the VAS at the last follow-up after WNA and other TCM treatments for KOA. There are 215 WNA-treated patients and 212 other TCM-treated patients. The analysis results yielded $\mathrm{P}=0.0009$ and $\mathrm{I}^{2}=76 \%$, indicating that there was heterogeneity between VAS at the last follow-up, so the random effects model was used for combined analysis. The combined effect size MD was
$-1.06,95 \%$ CI: -1.61 to -0.51 , as shown in Figure 7 . The overall effect size test was $\mathrm{Z}=3.78, \mathrm{P}=0.0002$, so the metaanalysis results suggested that the WNA treatment group was statistically different from the other TCM treatment groups in the last follow-up VAS.

\section{Publication bias}

The funnel plot was used to check the publication bias of the articles, including the effective rate and the last follow- 


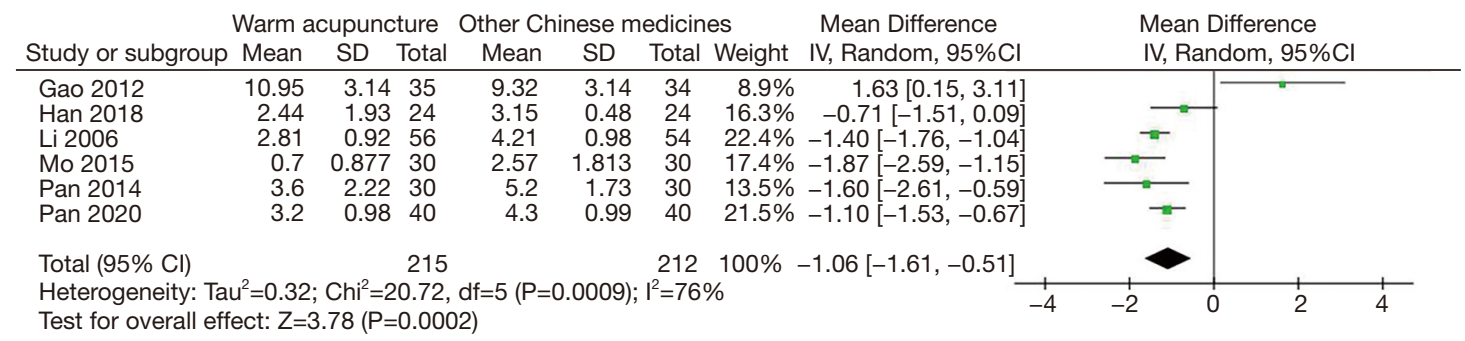

Figure 7 Forest plot of last follow-up VAS. Comparison of last follow-up VAS between the WNA group and the other TCM group. Statistical method: inverse variance of the random effects model (MD and 95\% CI). CI, confidence interval; VAS, visual analogue scale; WNA, warm needle acupuncture; TCM, traditional Chinese medicine; MD, mean difference.
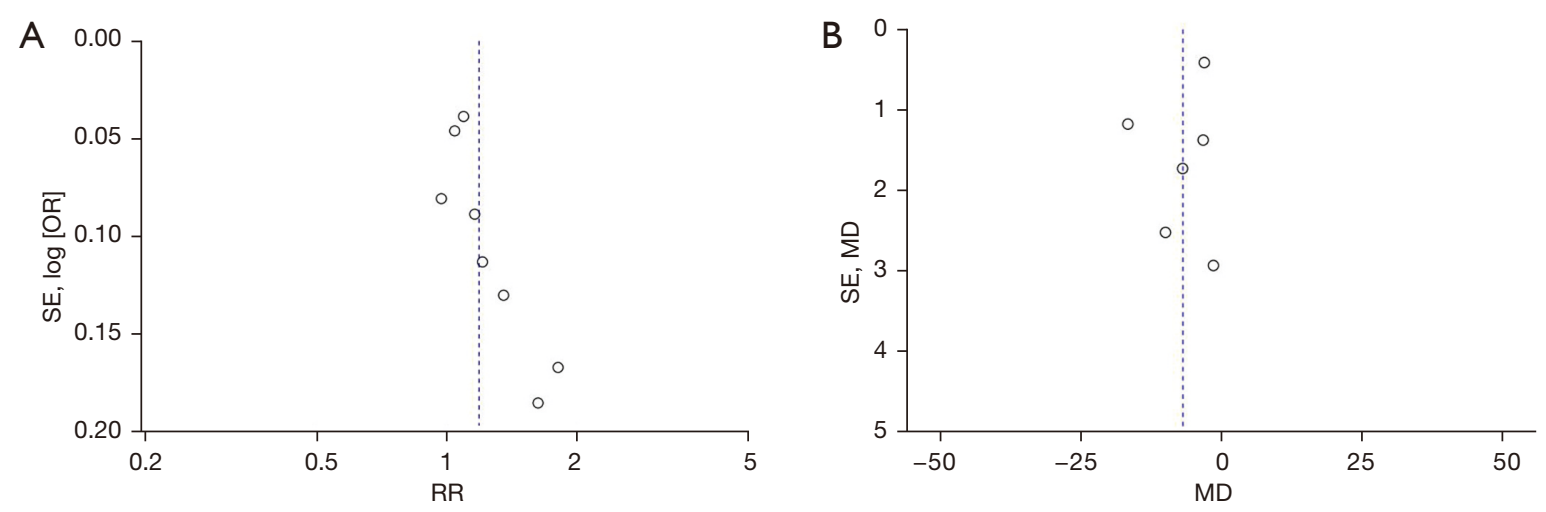

Figure 8 Funnel plot analysis of possible publication bias in subgroups. Effective rate (A) and last follow-up osteoarthritis index scale (B) funnel plot. SE, standard error of the mean; RR, relative risk; MD, mean difference.

up osteoarthritis index scale of WNA and other TCM treatments for KOA. It can be seen in Figure 8 that the funnel plot is asymmetrical, indicating that there may have been publication bias in both the effective rate and the last follow-up osteoarthritis index scale.

\section{Discussion}

Osteoarthritis is a common disease with many risk factors and complicated pathogenesis. The main risk factors include physical factors such as age, female gender, obesity, and family history, and mechanical factors such as local trauma and improper force lines (23). Clinically, the knee joint has the highest incidence of $\mathrm{OA}$, and KOA can cause body pain, joint movement dysfunction, and poor quality of life, which presents a huge economic burden to individuals and society. According to statistics, about $10-25 \%$ of people over 60 years old experience KOA $(3,24)$. However, at present, there is no consensus on the best treatment plan to improve the symptoms of KOA, because the conventional western medicine used to treat KOA is accompanied by side effects (25).

In TCM, KOA belongs to the category of "Bi Zheng", and the earliest written record of KOA can be found in the "Huang Di Nei Jing" $(26,27)$. The WNA is one of the main external treatment methods of TCM. The WNA treatment of KOA has significant advantages such as marked curative effect, few side effects, simple operation, and easy acceptance by patients (28). It stimulates the acupoints while warming the acupuncture points with the heat generated by moxibustion, which can better treat and prevent diseases (29-31). Studies have confirmed that WNA can regulate immune response, cell proliferation cycle, differentiation, and apoptosis through a variety of cell signal transduction pathways, so as to relieve the clinical symptoms of KOA (31,32).

There are many other treatment modalities in TCM, including herbal medicine, massage, acupuncture, electroacupuncture, cupping, and fumigation and washing. In order to understand the difference between WNA and 
other TCM techniques, this study specifically compared WNA treatment with other TCM treatment of KOA patients. The analysis results showed that compared with other TCM techniques, WNA had statistical differences in treatment efficiency, last follow-up osteoarthritis index, and last follow-up knee pain VAS, indicating that WNA has more advantages in treating KOA. However, the daily activities of the 2 groups of patients were similar, which may be related to the nature of the KOA disease, because these treatments can only alleviate the progression of the disease and relieve the pain of the patient, but cannot reverse the progression of the disease. When conservative treatments such as TCM or WNA are ineffective and seriously affect the quality of life of patients with KOA, knee replacement is an effective treatment measure.

The study highlighted the advantages of WNA, but also some limitations. First, the number of articles included in the meta-analysis was small, the sample size of cases was small, and it lacked representativeness. Second, the study failed to compare each TCM method one by one, which may have affected the true efficacy of WNA. Despite these limitations, this study systematically evaluated the effectiveness of WNA.

\section{Conclusions}

In summary, our comparative analysis of the curative effect of WNA and other TCM in the treatment of KOA patients shows that the overall curative effect of WNA is superior to that of other TCM modalities. However, further prospective RCTs are needed to compare WNA with other TCM modalities one by one, and further validate the therapeutic power of WNA.

\section{Acknowledgments}

Funding: None.

\section{Footnote}

Reporting Checklist: The authors have completed the PRISMA reporting checklist. Available at https://apm. amegroups.com/article/view/10.21037/apm-21-3972/rc

Conflicts of Interest: Both authors have completed the ICMJE uniform disclosure form (available at https://apm. amegroups.com/article/view/10.21037/apm-21-3972/coif). The authors have no conflicts of interest to declare.
Ethical Statement: The authors are accountable for all aspects of the work in ensuring that questions related to the accuracy or integrity of any part of the work are appropriately investigated and resolved.

Open Access Statement: This is an Open Access article distributed in accordance with the Creative Commons Attribution-NonCommercial-NoDerivs 4.0 International License (CC BY-NC-ND 4.0), which permits the noncommercial replication and distribution of the article with the strict proviso that no changes or edits are made and the original work is properly cited (including links to both the formal publication through the relevant DOI and the license). See: https://creativecommons.org/licenses/by-nc-nd/4.0/.

\section{References}

1. Dawson J, Linsell L, Zondervan K, et al. Impact of persistent hip or knee pain on overall health status in elderly people: a longitudinal population study. Arthritis Rheum 2005;53:368-74.

2. Palmer KT, Reading I, Calnan M, et al. Does knee pain in the community behave like a regional pain syndrome? Prospective cohort study of incidence and persistence. Ann Rheum Dis 2007;66:1190-4.

3. Michael JW, Schlüter-Brust KU, Eysel P. The epidemiology, etiology, diagnosis, and treatment of osteoarthritis of the knee. Dtsch Arztebl Int 2010;107:152-62.

4. Blagojevic M, Jinks C, Jeffery A, et al. Risk factors for onset of osteoarthritis of the knee in older adults: a systematic review and meta-analysis. Osteoarthritis Cartilage 2010;18:24-33.

5. McAlindon TE, Bannuru RR, Sullivan MC, et al. OARSI guidelines for the non-surgical management of knee osteoarthritis. Osteoarthritis Cartilage 2014;22:363-88.

6. London NJ, Miller LE, Block JE. Clinical and economic consequences of the treatment gap in knee osteoarthritis management. Med Hypotheses 2011;76:887-92.

7. Kim H, Shim I, Yi SH, et al. Warm needle acupuncture at Pungsi (GB31) has an enhanced analgesic effect on formalininduced pain in rats. Brain Res Bull 2009;78:164-9.

8. Wang Z, Wang J, Chan P. Treating type 2 diabetes mellitus with traditional chinese and Indian medicinal herbs. Evid Based Complement Alternat Med 2013;2013:343594.

9. Xiang Y, Guo Z, Zhu P, et al. Traditional Chinese medicine as a cancer treatment: Modern perspectives of ancient but advanced science. Cancer Med 2019;8:1958-75. 
10. Khan RMM, Chua ZJY, Tan JC, et al. From Pre-Diabetes to Diabetes: Diagnosis, Treatments and Translational Research. Medicina (Kaunas) 2019;55:546.

11. Phua WWT, Wong MXY, Liao Z, et al. An aPPARent Functional Consequence in Skeletal Muscle Physiology via Peroxisome Proliferator-Activated Receptors. Int J Mol Sci 2018;19:1425.

12. Si Y, Ma Y, Guo Y, et al. Efficacy and safety of Shaoyang Xibi decoction in patients with knee osteoarthritis: a multicenter, single-blind, randomized controlled trial. J Tradit Chin Med 2018;38:733-9.

13. Xu YK, Zhu FG, Feng EY, et al. External therapies of traditional Chinese medicine combined with sodium hyaluronate injected in articular cavity therapy on knee osteoarthritis: Meta-analysis. Zhongguo Zhong Yao Za Zhi 2018;43:1934-9.

14. Ou ZX, Jin JC, Huang D. Comparative Study on Effects of Combined Massage-Smouldering-Washing Therapy and Mini-invasive Surgery in Treating Knee Osteoarthritis. Zhongguo Zhong Xi Yi Jie He Za Zhi 2008;28:925-8.

15. Gao J, Ouyang BS, Zhang Y, et al. Comparison of the clinical therapeutic effects between electroacupuncture and warming needle moxibustion for knee osteoarthritis of kidney deficiency and marrow insufficiency pattern/ syndrome. Zhongguo Zhen Jiu 2012;32:395-8.

16. Li CD, Huang XY, Yang XG, et al. Observation on therapeutic effect of warming needle moxibustion on knee osteoarthritis of deficiency-cold type. Zhongguo Zhen Jiu 2006;26:189-91.

17. Han Y, Chen S, Pan X, et al. Clinical Study on Warming Needle Moxibustion for Treatment of Knee Osteoarthritis of Yang Deficiency and Cold Coagulation. Henan Traditional Chinese Medicine 2018;38:1096-9.

18. Mo Y. The clinical curative observation of needle warming moxibustion on knee osteoarthritis. Guangzhou: Guangzhou University of Chinese Medicine, 2015.

19. Pan L. Clinical observation of Yang deficiency Hanning Type of Knee Osteoarthritis with the warm acupuncture and electro-acupuncture. Jinan: Shandong University of Traditional Chinese Medicine, 2014.

20. Pan S, Wang Q, Zhou X. Observation on the curative effect of warming acupuncture in the treatment of knee osteoarthritis. Chinese Journal of General Practice 2020;18:1362-4.

21. Qu H, Wang R, Liu J. Clinical observation on warm needling in canicular days for knee osteoarthritis. Journal of Acupuncture and Tuina Science 2015;13:63-6.

22. Wu Y, Zhang J, Li S. Clinical observation on treatment of knee osteoarthritis by warm needling method. Journal of Acupuncture and Tuina Science 2009;7:349-51.

23. Hussain SM, Neilly DW, Baliga S, et al. Knee osteoarthritis: a review of management options. Scott Med J 2016;61:7-16.

24. Hinman RS, Campbell PK, Lawford BJ, et al. Does telephone-delivered exercise advice and support by physiotherapists improve pain and/or function in people with knee osteoarthritis? Telecare randomised controlled trial. Br J Sports Med 2020;54:790-7.

25. Li J, Li YX, Luo LJ, et al. The effectiveness and safety of acupuncture for knee osteoarthritis: An overview of systematic reviews. Medicine (Baltimore) 2019;98:e16301.

26. Liu J, Pan J, Wang Y, et al. Component analysis of Chinese medicine and advances in fuming-washing therapy for knee osteoarthritis via unsupervised data mining methods. J Tradit Chin Med 2013;33:686-91.

27. Yang M, Jiang L, Wang Q, et al. Traditional Chinese medicine for knee osteoarthritis: An overview of systematic review. PLoS One 2017;12:e0189884.

28. Ding MH, Zhang H, Li Y. A randomized controlled study on warming needle moxibustion for treatment of knee osteoarthritis. Zhongguo Zhen Jiu 2009;29:603-7.

29. Liu W, Fan Y, Wu Y, et al. Efficacy of AcupunctureRelated Therapy in the Treatment of Knee Osteoarthritis: A Network Meta-Analysis of Randomized Controlled Trials. J Pain Res 2021;14:2209-28.

30. Wei Y, Yuan N, Ding J, et al. Efficacy and safety of warm needle acupuncture in knee osteoarthritis: A protocol for systematic review and meta-analysis. Medicine (Baltimore) 2020;99:e23596.

31. Yang LP Wang M, Liu W, et al. Effects of warming-needle therapy on gene expression pathways in the patient with knee osteoarthritis of deficiency-cold syndrome. Zhongguo Zhen Jiu 2007;27:677-80.

32. Wang M, Liu L, Zhang CS, et al. Mechanism of Traditional Chinese Medicine in Treating Knee Osteoarthritis. J Pain Res 2020;13:1421-9.

(English Language Editor: J. Jones)

Cite this article as: Jin S, Guan X. A systematic review and meta-analysis of the comparative curative effects of warm acupuncture and other traditional Chinese medicines in the treatment of knee osteoarthritis. Ann Palliat Med 2022;11(2):708-716. doi: 10.21037/apm-21-3972 